\title{
Forced mode coupling in 2D complex plasmas
}

This content has been downloaded from IOPscience. Please scroll down to see the full text. 2016 EPL 11545002

(http://iopscience.iop.org/0295-5075/115/4/45002)

View the table of contents for this issue, or go to the journal homepage for more

Download details:

IP Address: 129.247.247.239

This content was downloaded on 13/12/2016 at 10:33

Please note that terms and conditions apply.

You may also be interested in:

Nonlinear regime of the mode-coupling instability in 2D plasma crystals

T. B. Röcker, L. Couëdel, S. K. Zhdanov et al.

Synchronization of particle motion in compressed two-dimensional plasma crystals

I. Laut, C. Räth, S. Zhdanov et al.

Interaction of two-dimensional plasma crystals with upstream charged particles

C.-R. Du, V. Nosenko, S. Zhdanov et al.

Wake turbulence observed behind an upstream "extra" particle in a complex (dusty) plasma

S. Zhdanov, C.-R. Du, M. Schwabe et al.

Laser manipulation ofparticles in dusty plasmas

A Melzer

Dynamical correlations and collective excitations of Yukawa liquids

Z Donkó, G J Kalman and P Hartmann

Detection of eddy current in the striation

V. Yu. Karasev, E. S. Dzlieva and S. I. Pavlov

PKE-Nefedov*:plasma crystal experiments on the International Space Station

Anatoli P Nefedov, Gregor

E Morfill, Vladimir E Fortov et al. 


\title{
Forced mode coupling in 2D complex plasmas
}

\author{
L. Coü̈del ${ }^{1}$, T. B. Röcker ${ }^{2}$, S. K. Zhdanov ${ }^{3}$, V. Nosenko $^{3}$, H. M. Thomas ${ }^{3}$ and A. V. Ivlev ${ }^{2}$ \\ 1 CNRS, Aix Marseille Université, PIIM, UMR 7345 - F-13397 Marseille, France \\ 2 Max Planck Institute for extraterrestrial Physics - D-85741 Garching, Germany \\ 3 Forschungsgruppe Komplexe Plasmen, Deutsches Zentrum für Luft und Raumfahrt - D-82234 Weßling, Germany
}

received 8 July 2016; accepted in final form 25 August 2016

published online 15 September 2016

PACS 52.27.Lw - Dusty or complex plasmas; plasma crystals

\begin{abstract}
It is demonstrated experimentally that the plasma-wake-mediated resonant coupling of the longitudinal in-plane and out-of-plane collective motion in two-dimensional plasma crystals can be induced by applying an external forcing. The experimental observations are supported by numerical analysis of the forced collective dynamics of particles with the wake-mediated interactions.
\end{abstract}

\section{editor's choice Copyright (c) EPLA, 2016}

Introduction. - Two-dimensional (2D) complex plasmas $[1,2]$ are often used as model systems to study at the kinetic (particle) level generic phenomena occurring in liquids and crystals [3,4] such as phase transitions, wave propagation, dislocation dynamics, plastic deformation [5-8]. In a radio-frequency (rf) discharge, microparticles are levitating in the sheath where an inhomogeneous vertical electric field exerts an electric force able to balance the gravity $[9,10]$, and to ensure stiff confinement of the monolayer. On the other hand, the fieldinduced ion stream is focussed downstream of each particle and thus creates a perturbed region called the "plasma wake". Wakes exert an attractive force on the neighbouring particles and make the particle pair interactions nonreciprocal [11-14].

Generally, in 2D complex plasma crystals, three wave modes can be sustained: two in-plane modes - longitudinal (compressional) and transverse (shear) [15], and one out-of-plane mode [16]. When the crystal is sufficiently dense and/or the vertical confinement is sufficiently weak, the out-of-plane wave mode can cross the in-plane longitudinal mode (usually this occurs at the border of the first Brillouin zone). Due to the non-reciprocal wake forces, the crossing is accompanied by the formation of the hybrid mode which has a positive growth rate, i.e., energy is transferred from the flowing plasma to the crystal. This can trigger the mode coupling instability (MCI) $[17,18]$ and the melting of the crystalline monolayer [19-22]. The hybrid mode has distinct fingerprints: critical angular dependence (the formation of the hybrid mode and the onset of the mode coupling instability depend on the direction of the wave vector compared to the lattice main axis - see inset of fig. 1), a mixed polarisation,

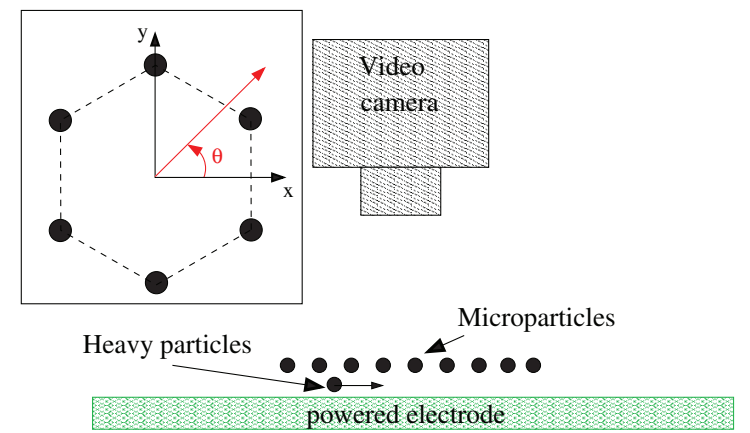

Fig. 1: (Colour online). Sketch of the experimental setup. Microparticles are confined above the rf powered electrode. The Mach cone and subsonic lateral wakes are triggered by heavy particles moving under the crystalline monolayer. The inset shows elementary cell of the hexagonal lattice and the frame of reference chosen in this paper, the orientation angle $\theta$ of the wave vector $\mathbf{k}$ is measured from the $x$-axis.

confinement and dust density thresholds [19], synchronisation of the particle motion [23].

In a recent article [20], it was shown theoretically that wake-induced mode coupling is possible in both crystalline and liquid complex plasmas. Remarkably, in liquid complex plasmas, the confinement and density thresholds (which are important features of the MCI in 2D crystals) disappear, and the instability growth rate is higher.

In this letter, we demonstrate experimentally that the wake-mediated mode coupling can also be induced by forcing in-plane longitudinal waves. The resonances between these forced modes and the out-of-plane eigenmode reveal characteristic fingerprints of the hybrid mode. 
Table 1: Experimental parameters. Parameters of the 2D crystal have been extracted from experimental data by simultaneously fitting the low-wave-number part $(k \Delta<2.5)$ of the longitudinal and transverse spectra with the theoretical dispersion relation obtained for pure Yukawa interactions [22]. The errors for $Q$ and $\kappa$, obtained from the nonlinear least squares fit (95\% confidence interval), are $\simeq 6 \%$ and $\simeq 22 \%$, respectively. The vertex velocities are the averaged values in the analysed field of view, obtained after tracking.

\begin{tabular}{llccc}
\hline Parameter & Symbol & Unit & Exp. & Simulation \\
\hline Argon pressure & $p$ & $\mathrm{~Pa}$ & 0.42 & - \\
RF power & $P_{w}$ & $\mathrm{~W}$ & 20 & - \\
Particle diameter & $2 a$ & $\mu \mathrm{m}$ & $9.19 \pm 0.14$ & - \\
Averaged interparticle distance & $\Delta$ & $\mu \mathrm{m}$ & 550 & 550 \\
Longitudinal dust-lattice wave speed & $C_{L}$ & $(\mathrm{~mm} / \mathrm{s})$ & $44.7 \pm 5.1$ & 47.5 \\
Transverse dust-lattice wave speed & $C_{T}$ & $(\mathrm{~mm} / \mathrm{s})$ & $8.2 \pm 1.5$ & 8.3 \\
Debye length & $\lambda$ & $(\mu \mathrm{m})$ & 733 & 733 \\
Screening parameter & $\kappa=\Delta / \lambda$ & & 0.97 & 0.75 \\
Particle charge in electron charges & $Q$ & $(e)$ & 21460 & 20000 \\
Vertical resonance frequency & $f_{v}$ & $(\mathrm{~Hz})$ & $24 \pm 1$ & 22 \\
Mach cone vertex velocity & $v_{M C}$ & $(\mathrm{~mm} / \mathrm{s})$ & $60.4 \pm 4.5$ & - \\
Subsonic lateral wake vertex velocity & $v_{S W}$ & $(\mathrm{~mm} / \mathrm{s})$ & $42.3 \pm 1.4$ & - \\
\hline
\end{tabular}

Experimental setup. - The experimental setup used in this study has been extensively described in previous publications $[19,21,24]$. Experiments were performed in a (modified) GEC chamber, in a capacitively coupled rf glow discharge at $13.56 \mathrm{MHz}$. The argon pressure $p$ was between $0.4 \mathrm{~Pa}$ and $1 \mathrm{~Pa}$ and the forward $\mathrm{rf}$ power $P$ was between $5 \mathrm{~W}$ and $20 \mathrm{~W}$ (see fig. 1). A horizontal monolayer, up to $60 \mathrm{~mm}$ in diameter, was formed by levitating melamine-formaldehyde particles with a diameter $2 a=9.19 \pm 0.14 \mu \mathrm{m}$ in the plasma sheath above the lower rf electrode. The dust particle cloud was illuminated by two laser sheets: a vertical one and a horizontal one. The particles were imaged through a window at the top of the chamber by a Photron FASTCAM 1024 PCI camera at a speed of 250 frames per second. The particle horizontal coordinates, $x$ and $y$, and the respective velocities, $v_{x}$ and $v_{y}$, were then extracted with sub-pixel resolution in each frame by using a standard particle tracking technique [25]. The particle vertical velocities $v_{z}$ were deduced by analysing the scattered-light intensity variations [26]. An additional side-view camera (Basler Ace ACA640-100GM or Photron FASTCAM 1024 PCI) was used to check that we were indeed working with a single layer of particles.

Results. - To launch waves in the plasma crystal, we used the wakes of fast-moving extra particles beneath the main layer. This method was inspired by Samsonov et al. [27]. In order to create the Mach cone (MC) [27] and the subsonic lateral wakes (SW) [28], the particle cloud was not completely purified. A few (heavier) particles were present beneath the crystalline monolayer (see fig. 1), with velocities above (MC) or below (SW) the longitudinal sound speed in the lattice (see table 1). The moving heavy particle lifted up the microparticles directly above it and compressed the monolayer in the direction of
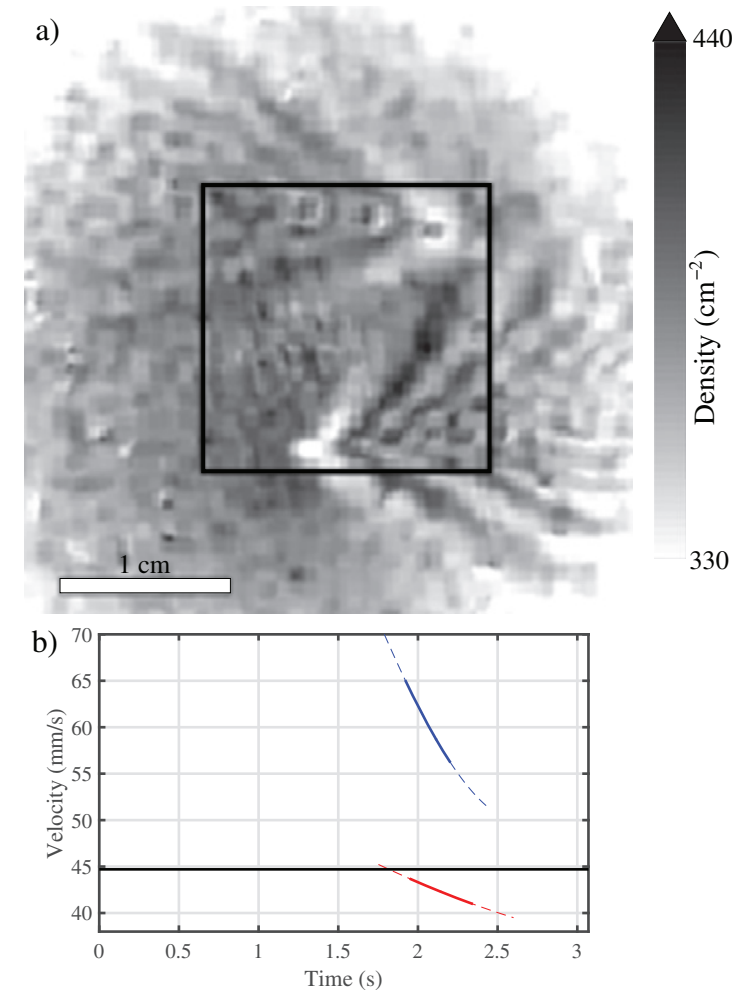

Fig. 2: (Colour online) a) Map of the particle number density calculated at the inverse area of the respective Voronoi cells (coded from white to black). The black frame is the field of view used to compute of the current spectra. The spatial scale is shown by the horizontal bar in the bottom left corner. Note the Mach cone (MC) at the top and the subsonic lateral wake (SW) at the bottom. b) Velocities of the heavy particles generating MC (blue) and SW (red) in the time span used for the calculation of the current fluctuation spectra. Bold lines are when the heavy particles are in the analysed field of view. 
its motion. While these excitations had three-dimensional structure [24], the launched in-plane waves were much stronger than the out-of-plane ones (the launched in-plane waves had larger amplitudes and greater spatial extent than the out-of-plane waves). Due to the strongly-coupled nature of the crystalline state, the created excitations were first compressive and then rarefactive. MC and SW consisted of two waves (the wing) propagating at a given angle compared to the lattice orientation (see fig. 2). The $\mathrm{MC}$ wings were straight while the SW wings were bent. These forced oscillations were thus used to investigate the induced mode-coupling resonances.

The parameters of the crystal (see table 1) were such that the crossing of the in-plane longitudinal mode with the out-of-plane mode was very shallow and only visible at an angle of $0^{\circ}$ (or $180^{\circ}$ ) compared to the lattice main axis $[19,22]$. This is well evidenced in fig. 3(a) and (c) where traces of mixed polarisation and hot spots are visible near the border of the first Brillouin zone. The heavy particles moved in a straight line at a given angle compared to the lattice main axis. Consequently, MC and SW traces in the in-plane longitudinal spectra were angledependent. These traces are clearly visible in figs. 3(a) and (c) as straight lines in the low- $k$ part of the longitudinal fluctuation spectra. It should be noted that the forced mode branches are clearly detached from the thermal wave modes for normalised wave number $k \Delta \gtrsim 1$, where $\Delta$ is the mean interparticle distance. The resonant coupling of the forced in-plane modes (lower branches) and the out-of-plane eigenmode (upper branch) is revealed by the induced hot spots occurring at the respective crossing points (fig. 3). These spots are perfectly aligned with the low- $k$ traces visible in the in-plane spectra. The forced coupling is observed, as it has to be expected, both in the in-plane and out-of-plane spectra. The traces of the mixed polarisation, well recognisable in the longitudinal fluctuation spectra, are signatures of wake-mediated mode coupling [19]. Therefore, their enhancement at the crossing points with the acoustic excitations, seen in fig. 3(a), (c), (d), (f) as bright spots in the spectra, is a clear manifestation of a forced wake-mediated resonance. It is important to note also that a direct resonant excitation of the MC and SW waves caused by the essentially three-dimensional character of the perturbation, even if significantly weaker, could not be ruled out. For example, the relatively weak forced vertical oscillations observed in ref. [24], despite their evident resonant nature, were of this kind. The hot spot positions are also angle-dependent (see supplemental movie Supplemental. avi ${ }^{1}$ ).

Discussion. - In 2D complex plasmas, the wakemediated coupling between the collective in-plane and

\footnotetext{
${ }^{1}$ From the left to the right: out-of-plane, in-plane longitudinal, and in-plane transverse particle current fluctuation spectra as a function of the propagation angle $\theta . \theta$ is given compared to the lattice main axis (see inset of fig. $1 ; \theta=0^{\circ}$ corresponds to a $-14.5^{\circ}$ rotation of the video frames). Note the angular dependence of the MC and SW traces. (The video has been tested with VLC.)
}

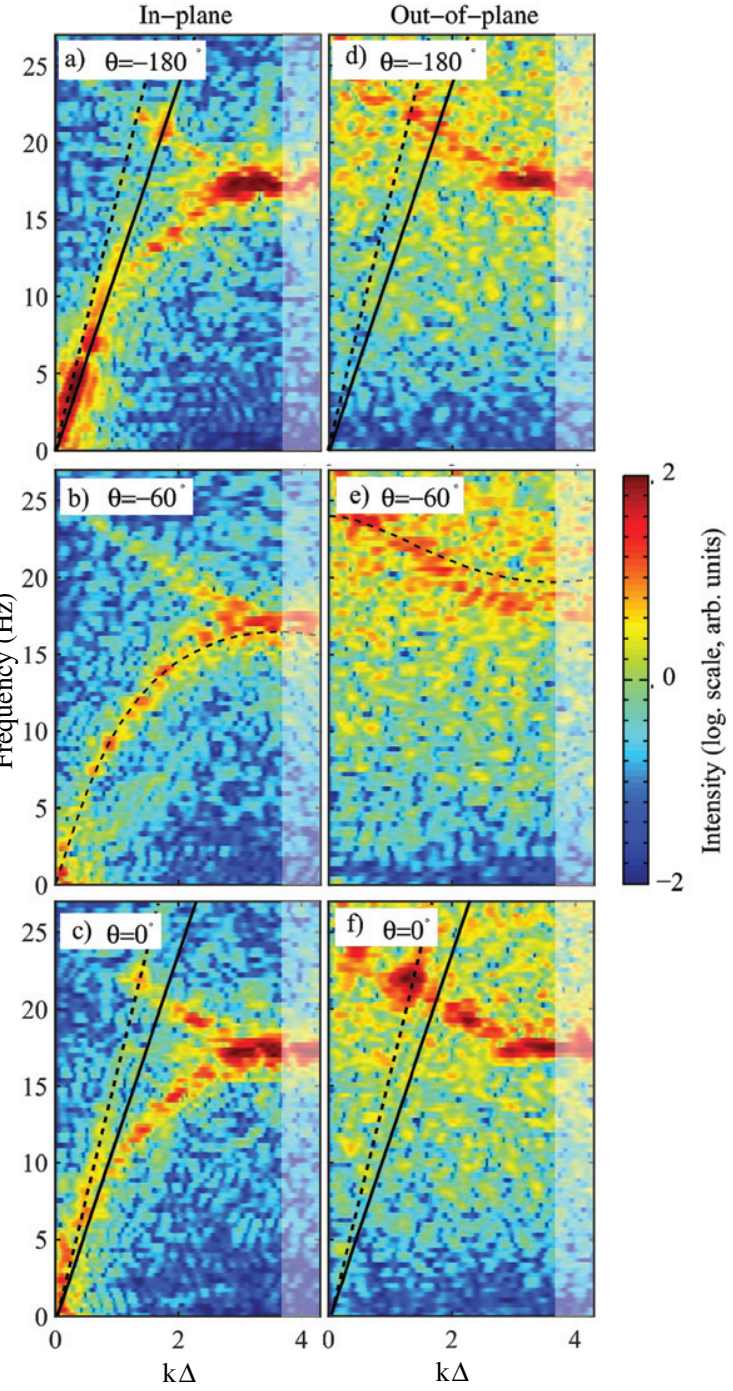

Fig. 3: (Colour online) Resonant coupling with ((a), (d)) the subsonic lateral wake $(\mathrm{SW})$ and with $((\mathrm{c}),(\mathrm{f}))$ the Mach cone (MC). Spectra in a direction exhibiting no resonance are given in (b), (e). (a)-(c) and (d)-(f) are the in-plane longitudinal and out-of-plane particle velocity fluctuation spectra, respectively. Only the first Brillouin zone is shown, the beginning of the second zone is shaded. In (b), (e), the fitted longitudinal and outof-plane dispersion curves are shown (thin dashed lines). Note that the fit was performed simultaneously for the in-plane longitudinal and transverse spectra and not for the out-of-plane spectrum. SW ((a), (d)) and MC ((c), (f)) primarily generate in-plane collective motion - the forced modes underlined, respectively, by the bold-solid and bold-dashed lines are clearly visible in (a), (c). The resonant coupling of the forced in-plane modes (lower branches) with the out-of-plane (upper branch) eigenmode, occurring at the respective crossing points, is revealed by the induced hot spots. In the most unstable direction $\theta=-180^{\circ}$ and $\theta=0^{\circ}$ (see the inset of fig. 1), the bright spots close to the border of the first Brillouin zone indicate the formation of the regular hybrid mode (due to the crossing of the eigenmodes) $[19,22]$. The angle $\theta$ is given compared to the lattice main axis $\left(\theta=0^{\circ}\right.$ corresponds to a $-14.5^{\circ}$ rotation of the video frames). 


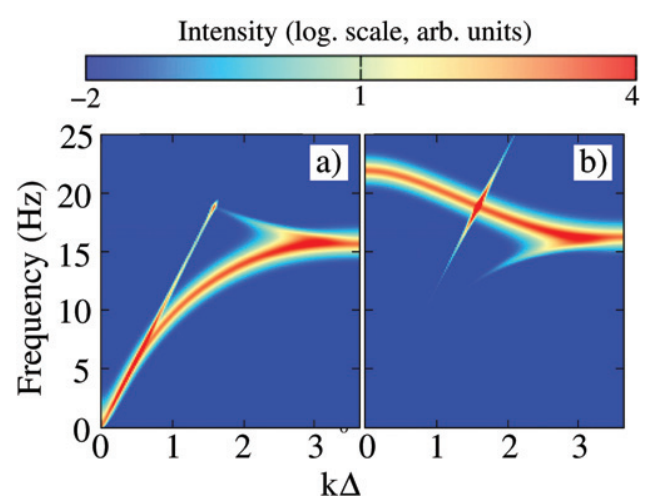

Fig. 4: (Colour online) In-plane longitudinal (a) and out-ofplane (b) fluctuation spectra, numerically calculated for the conditions of the Mach cone experiment and representing, respectively, the spectra shown in fig. 3(c) and (f). The acoustic excitation (straight line) is produced by a test charge moving underneath a crystalline monolayer along the direction $\theta=0^{\circ}$, to mimic the Mach cone generated by a supersonic projectile particle.

out-of-plane motion of particles is driven by the nonreciprocity of the interparticle interactions. This is a very generic phenomenon which can occur in any manyparticle system where the action-reaction symmetry of the effective interactions is broken due to the presence of a non-equilibrium environment [29]: examples include optical [30] and diffusiophoretic [31] forces between colloids, the effective interactions under solvent or depletant flow [32-34], shadow forces in 3D complex plasmas [35], etc. It is important to stress that wake-mediated coupling is a pure dynamical phenomenon, and therefore occurs irrespectively of whether the collective particle motion represents a wave eigenmode or is driven externally. In particular, this implies that the mode-coupling instability in 2D complex plasmas can also develop due to resonant coupling between the out-of-plane eigenmode and the in-plane forced mode. For the presented experiment, the induced resonances were clearly seen through the enhancement of the SW and MC traces in the out-of-plane spectra (red spots in fig. 3(d) and (f)), and also visible in the traces of the out-of-plane mode in the in-plane spectra (due to mixed polarisation, see fig. 3(a) and (c)).

Theoretical calculations of the "forced" resonance, presented in fig. 4 demonstrate good agreement with the experimental observations. To obtain theoretical spectra of fig. 4, the Fourier-transformed equations of motion for the individual particles were considered. Herein, thermal motion was modelled by uncorrelated stochastic forces (Gaussian white noise) balanced on average by dust-neutral friction, in accordance with the fluctuationdissipation-theorem [36]. In the simulation we also observed the characteristic features peculiar to the "conventional" mode-coupling instability, such as traces of mixed polarisation and hot spots at the resonance frequencies. The interaction between the sonic "heavy" particle (travelling at a fixed distance below the monolayer at an angle of $0^{\circ}$ compared to the main lattice direction) and the monolayer was described by Yukawa/point-wake model [37]. The resulting particle motion gives the Mach cone pattern seen in fig. 4(a), (b).

Conclusion. - To conclude, we have experimentally demonstrated that wake-mediated resonant mode coupling can be induced in a two-dimensional plasma crystal by exciting (predominantly) longitudinal waves in the crystals (using heavy particles travelling under the crystalline layer). The observations are supported by calculations of the forced wake-induced resonance based on the theory of the mode-coupling instability in complex plasma crystals $[19,22,37]$. The obtained results demonstrate the fundamental effect of the wake-mediated mode coupling in 2D and quasi-2D plasma crystals.

$* * *$

This work was partially supported by the French-German PHC PROCOPE Program (Project No. 35325NA/57211784) and by the European Research Council under the European Union's Seventh Frame-work Programme FP7 2007-2013 (ERC Grant Agreement No. 267499).

\section{REFERENCES}

[1] Chu J. and I Lin, Physica A: Stat. Mech. Appl., 205 (1994) 183.

[2] Thomas H. M. and Morfill G. E., Nature, 379 (1996) 806.

[3] Glaser M. A. and Clark N. A., Melting and Liquid Structure in Two Dimensions, Vol. 83 (John Wiley \& Sons, Inc.) 2007, Chapt. 7, pp. 543-709.

[4] Ivlev A., Löwen H., Morfill G. and Royall C. P., Complex Plasmas and Colloidal Dispersions: ParticleResolved Studies of Classical Liquids and Solids (World Scientific, Singapore) 2012.

[5] Chiang C.-H. and I Lin, Phys. Rev. Lett., 77 (1996) 647.

[6] Farouki R. T. and Hamaguchi S., Appl. Phys. Lett., 61 (1992) 2973.

[7] Hebner G. A., Riley M. E., Johnson D. S., Ho P. and Buss R. J., Phys. Rev. Lett., 87 (2001) 235001.

[8] Kalman G., Rosenberg M. and DeWitt H. E., Phys. Rev. Lett., 84 (2000) 6030.

[9] Brattli A. and Havnes O., J. Vac. Sci. Technol. A, 14 (1996) 644.

[10] Samarian A. A. and James B. W., Phys. Lett. A, 287 (2001) 125.

[11] Ishihara O. and Vladimirov S. V., Phys. Plasmas, 4 (1997) 69.

[12] Nunomura S., Misawa T., Ohno N. and Takamura S., Phys. Rev. Lett., 83 (1999) 1970.

[13] Melzer A., Schweigert V. A. and Piel A., Physica Scripta, 61 (2000) 494.

[14] Hebner G. A. and Riley M. E., Phys. Rev. E, 69 (2004) 026405.

[15] Qiao K. and Hyde T. W., Phys. Rev. E, 68 (2003) 046403. 
[16] Liu B., Avinash K. and Goree J., Phys. Rev. Lett., 91 (2003) 255003.

[17] Ivlev A. V. and Morfill G., Phys. Rev. E, 63 (2001) 016409.

[18] Ivlev A. V., Konopka U., Morfill G. and Joyce G., Phys. Rev. E, 68 (2003) 026405.

[19] Couëdel L., Zhdanov S. K., Ivlev A. V., Nosenko V., Thomas H. M. and Morfill G. E., Phys. Plasmas, 18 (2011) 083707.

[20] Ivlev A. V., Zhdanov S. K., Lampe M. and Morfill G. E., Phys. Rev. Lett., 113 (2014) 135002.

[21] Röcker T. B., Couëdel L., Zhdanov S. K., Nosenko V., Ivlev A. V., Thomas H. M. and Morfill G. E., EPL, 106 (2014) 45001.

[22] Zhdanov S. K., Ivlev A. V. and Morfill G. E., Phys. Plasmas, 16 (2009) 083706.

[23] Couëdel L., Zhdanov S., Nosenko V., Ivlev A. V., Thomas H. M. and Morfill G. E., Phys. Rev. E, 89 (2014) 053108.

[24] Couëdel L., Samsonov D., Durniak C., Zhdanov S., Thomas H. M., Morfill G. E. and Arnas C., Phys. Rev. Lett., 109 (2012) 175001.

[25] Rogers S. S., Waigh T. A., Zhao X. and Lu J. R., Phys. Biol., 4 (2007) 220.
[26] Couëdel L., Nosenko V., Zhdanov S., Ivlev A. V., Thomas H. M. and Morfill G. E., Phys. Rev. Lett., 103 (2009) 215001.

[27] Samsonov D., Goree J., Thomas H. M. and Morfill G. E., Phys. Rev. E, 61 (2000) 5557.

[28] Nosenko V., Goree J., Ma Z., Dubin D. and Piel A., Phys. Rev. E, 68 (2003) 056409.

[29] Ivlev A. V., Bartnick J., Heinen M., Du C.-R., Nosenko V. and Löwen H., Phys. Rev. X, 5 (2015) 011035 .

[30] Dholakia K. and Zemánek P., Rev. Mod. Phys., 82 (2010) 1767.

[31] Soto R. and Golestanian R., Phys. Rev. Lett., 112 (2014) 068301.

[32] Khair A. S. and Brady J. F., Proc. R. Soc. London, Ser. A, 463 (2007) 223.

[33] Mejia-Monasterio C. and Oshanin G., Soft Matter, 7 (2011) 993.

[34] Sriram I. and Furst E. M., Soft Matter, 8 (2012) 3335.

[35] Tsytovich V. N., Phys. Usp., 40 (1997) 53.

[36] Röcker T. B., Ivlev A. V., Zhdanov S. K. and Morfill G. E., Phys. Rev. E, 89 (2014) 013104.

[37] Röcker T. B., Ivlev A. V., Kompaneets R. and Morfill G. E., Phys. Plasmas, 19 (2012) 033708. 